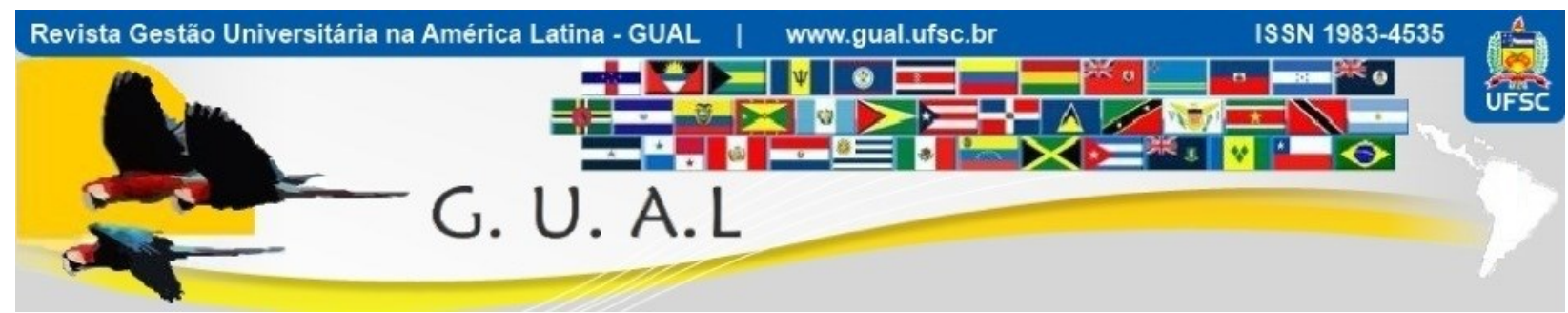

DOI: http://dx.doi.org/10.5007/1983-4535.2015v8n3p204

\title{
ENVOLVIMENTO DOS ALUNOS DE CURSOS DE GRADUAÇÃO EM ADMINISTRAÇÃO: APLICAÇÃO DE ESCALA MULTIDIMENSIONAL
}

INVOLVEMENT OF STUDENTS OF BUSINESS ADMINISTRATION GRADUATION COURSES: APPLICATION OF A MULTIDIMENSIONAL SCALE

Fernanda Pauletto D'Arrigo, Mestranda

Universidade de Caxias do Sul - UCS

fernanda.darrigo@gmail.com

Cláudia Prantz, Mestre Universidade de Caxias do Sul - UCS

cprantz@gmail.com

Márcio José Migliavacca, Mestrando

Universidade de Caxias do Sul - UCS marcio@rexfort.com.br

Cíntia Paese Giacomello, Doutora Universidade de Caxias do Sul - UCS cintia.paese@gmail.com

Pelayo Munhoz Olea, Doutor Universidade de Caxias do Sul - UCS pelayo.olea@gmail.com

Recebido em 14/dezembro/2014

Aprovado em 03/junho/2015

Sistema de Avaliação: Double Blind Review

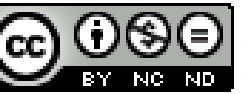

Esta obra está sob uma Licença Creative Commons Atribuição-Uso. 


\title{
RESUMO
}

Dos mais de sete milhões de universitários dos cursos de graduação no Brasil, mais de 18\% optam pelo curso de Administração. Em meio a concorrência dos cursos de graduação em Administração, o envolvimento individual do aluno interfere no processo de formação do profissional. Desta forma, o objetivo deste estudo é estudar o envolvimento dos alunos de Administração sob as dimensões que interferem no processo de aprendizagem, sendo elas: cognitiva, afetiva, comportamental e agenciativa relatadas por Veiga (2013). O estudo acontece em três diferentes instituições que oferecem ensino superior em Administração na cidade de Caxias do Sul, o que torna possível a comparação do envolvimento dos alunos em diferentes instituições de ensino superior em Administração. $\mathrm{O}$ estudo quantitativo descritivo, contou com uma amostra total de 137 respondentes para a realização da análise estatística, na qual concluiu que em três das quatro dimensões analisadas apresentaram diferenças significativas entre as instituições analisadas. A dimensão agenciativa, na qual o aluno busca enriquecer o aprendizado de forma intencional e proativa foi a que apresentou a maior divergência entre as instituições.

Palavras-Chaves: Envolvimento Acadêmico. Graduação em Administração. Processo de Aprendizagem. Análise Estatística.

\begin{abstract}
In Brazil there are over than seven million students of graduation courses in Brazil. More than $18 \%$ of these students choose for the Business Administration course. In the midst of competition from the Business Administration graduation courses, the individual student plays an important role in the student process of professional education. In this way, the aim of this study is to understand the involvement of students of Business Administration by dimensions (cognitive, affective, behavioral and agenciative) that affect the learning process as reported by Veiga (2013). The study was carried out in three different higher education institutions in business administration in the city of Caxias do Sul. The difference between institutions made possible to compare the involvement of students of different institutions of Business Administration. The quantitative approach included a total sample of 137 respondents to the statistical analysis, concluding that in three of the four dimensions analyzed showed significant differences between the institutions analyzed. The agenciative dimension in which the student aims to enrich the learning intentionally and proactively presented the most divergence between the institutions.
\end{abstract}

Key-Words: Academic Engagement. Graduation in Business Administration. Learning Process. Statistic Analysis. 


\section{INTRODUÇÃO}

Segundo a Associação Brasileira de Estágios (2012) dos mais de sete milhões de universitários regularmente matriculados em cursos de graduação no Brasil, mais de um milhão e trezentos mil alunos (18,8\%), optam por cursar Administração. No ano de 2012, o curso de Administração foi o mais procurado do país, totalizando 833.042 matrículas em 2012 (MEC, 2012). Em meio a demanda por cursos de ensino superior em Administração, pode-se perceber um aumento de instituições quais oferecem essa formação acadêmica. Em 2014, o Brasil conta com 1.554 cursos de Administração (bacharelado) regulamentados pelo Ministério da Educação. Esses cursos são oferecidos aos alunos na modalidade presencial ou a distância. Só no estado do Rio Grande do Sul, existem 115 cursos de Administração (MEC, 2014). Ainda segundo o Ministério da Educação (2014) a cidade de Caxias do Sul, no Rio Grande do Sul, com a população estimada em 435 mil habitantes, conta com 18 instituições de ensino superior que oferecem o curso de administração. Destas são 10 destas de ensino presencial e 8 instituições com ensino somente a distância.

Pensando nas diferentes opções de instituições que os alunos podem optar para cursar ensino superior em Administração, pode-se ressaltar o papel que o envolvimento individual do aluno tem na carreira acadêmica. Para Pascarella e Terenzini (2005) o envolvimento do estudante durante o curso de graduação é o determinante crítico para o sucesso acadêmico do aluno e da universidade. Segundo Felicetti e Morosini (2010) o comprometimento individual na aprendizagem, dá relevância na maneira de como o aluno aprende, e tem direta relação com a variedade e a intensidade dos meios de utilizados para a aprendizagem. Fior, Mercuri e Silva (2013) tratam o envolvimento acadêmico dos alunos de curso de graduação proporciona ricas oportunidades de aprendizado e desenvolvimento para o estudante, entretanto destacam que as instituições têm um papel ímpar no envolvimento acadêmico dos seus alunos, por meio do suporte para a viabilização das atividades de aprendizagem.

Desta forma, o objetivo deste estudo é estudar o envolvimento dos alunos dos cursos de Administração de três diferentes instituições da cidade de Caxias do Sul. As dimensões do envolvimento dos alunos foram selecionadas a partir do trabalho de Veiga (2013) for se tratarem de dimensões as quais interferem no processo de aprendizagem dos alunos em quatro dimensões: cognitiva, afetiva, comportamental e agenciativa. Para entender o papel da instituição de ensino no envolvimento do aluno com o curso, foram coletadas informações de três diferentes instituições de ensino superior em Administração na cidade de Caxias dos Sul. 
Por meio de análises estatísticas univariável e multivariável foi possível identificar os pontos divergentes e semelhantes entre as instituições pesquisadas e as dimensões de envolvimento do aluno analisadas.

\section{ENVOLVIMENTO ACADÊMICO DOS ALUNOS}

O envolvimento individual com as atividades relevantes para a aprendizagem se relacionam ao comprometimento deste aluno com a aprender (ENGERS e MOROSINI, 2007) Neste sentido Felicetti e Morosini (2010) tratam que o comprometimento e o envolvimento do aluno com a aprendizagem estão relacionados aos objetivos e aspirações individuais, e assim acaba criando o sentido de equilíbrio entre o querer e o fazer. Ambos os pontos de vistas seguem a linha de Austin (1984) que acredita que o envolvimento acadêmico acaba por novos conhecimentos, atitudes, crenças e valores que tem efeito no futuro profissional. Entretanto o envolvimento exige investimentos de recursos físicos e psicológicos por parte dos alunos.

Veiga (2013) construiu uma escala tratando o envolvimento dos alunos em idade escolar em quatro dimensões: cognitiva, afetiva, comportamental e agenciativa. Segundo o autor existe uma relação do aluno em dimensões específicas que afetam o desempenho dos alunos.

\subsection{DIMENSÃO COGNITIVA}

$\mathrm{O}$ envolvimento cognitivo dos alunos se refere às estratégias cognitivas que eles empregam na aprendizagem (WALKER, GREENE E MANSELL, 2006; REEVE E TENG, 2011; LAM et al. 2012). Walker, Greene e Mansell (2006) trazem a motivação intrínseca dos alunos como uma tendência de se envolver em tarefas por achar interessante e agradável. Os alunos que apresentam maior motivação intrínseca tendem a persistir em problemas difíceis e aprender com os erros (WALKER, GREENE e MANSELL, 2006). Além disso, a motivação intrínseca é central para o processo de integração, através do qual os elementos de conhecimento interno existentes, integrados com novos conhecimentos (NILSEN, 2009). Esta motivação intrínseca pode ter influência positiva no comportamento (MILLER, 1988).

Para Lam et al. (2012) os alunos podem utilizar estratégias de processamento de aprendizagem de maneira profunda ou superficial. Nas estratégias profundas a aprendizagem está associada à elaboração cognitiva do material a ser aprendido enquanto que as estratégias de processamento superficial envolvem memorização, ensaio básico, e outros tipos de 
envolvimento superficiais com o novo material. Os alunos que se envolvem em processamento cognitivo profundo tendem a ter uma melhor compreensão e retenção de materiais de aprendizagem significativa.

Para Veiga (2013) essa dimensão assenta no processamento da informação, com procura de relações, gestão da informação e elaboração de planos de execução. Para a avaliação deste constructo se levou em consideração como os alunos associam as teorias às práticas, ou como os hábitos dos alunos de rever as anotações, montar seus próprios exemplos e modelos, por exemplo, os faz melhor compreender as teorias e a melhorar seu desempenho.

\subsection{DIMENSÃO AFETIVA}

A dimensão afetiva refere-se aos sentimentos dos alunos sobre as atividades do processo de aprendizagem, levando em consideração o contexto social que as pessoas estão inseridas, a percepção sobre si mesmo resultado em ações ou de envolvimento ou de desafeição (CONNELL e WELLBORN, 1991).

Segundo Lam et al. (2011) alunos com alto envolvimento afetivo desfrutam aprender e tendem a gostar mais de frequentar as aulas. Os sentimentos criados sobre as atividades de aprendizagem são reflexos de motivação intrínseca, enquanto que os sentimentos sobre a instituição de ensino são uma manifestação de apego ao local. Quando a ideia de consideração e sentimento pela instituição que frequentam é abordada, é importante destacar Finn (1989), que relata que os alunos que se identificam com a instituição têm um conceito interno de pertencimento perceptível de que faz parte do ambiente escolar e que a instituição constitui uma parte importante de sua própria experiência, o que cria um senso de pertencimento à instituição.

No instrumento de Veiga (2013) o item com maior significância nesta dimensão é “A minha instituição é um lugar onde me sinto integrado (a)", já que o conteúdo dos itens tem a ver com a ligação à escola, em que a amizade, recebida e praticada, é saliente, bem como o sentido de inclusão e pertença à instituição.

\subsection{DIMENSÃO COMPORTAMENTAL}

Segundo Archambault et al. (2009) o constructo comportamental reflete diretamente nas dimensões cognitivas e nas características psicológicas atribuídas às questões básicas de participação e de conformidade dos alunos. De acordo com Lam et al. (2011) a dimensão 
comportamental refere-se à participação dos alunos em aprender, sua motivação nas ações realizadas e nos comportamentos diretamente ligados ao contexto, neste caso a relação aluno e professor (SKINNER e BELMONT, 1993).

Ao falar da dimensão comportamental, Veiga (2013) usou indicadores específicos de conduta dos alunos em ambientes escolares, tais como perturbar intencionalmente as aulas, o ser incorreto com os professores, o estar distraído nas aulas e, ainda, o faltar às aulas. Com esses indicadores o autor buscou mensurar em uma dimensão o comportamento dos alunos na sala de aula.

\subsection{DIMENSÃO AGENCIATIVA}

Além das dimensões comportamental, emocional/comportamental e cognitiva, Reeve e Tseng (2011) acrescentam a importância do aluno no processo da aprendizagem. Segundo os autores, o envolvimento do aluno durante as atividades de aprendizagem é importante como resultado educacional do progresso dos alunos e realização acadêmica. Com isso, eles definem o engajamento agenciativo como a contribuição construtiva dos alunos no fluxo de instruções do que recebem. Ou seja, o processo em que os alunos de forma intencional e próativa buscam personalizar e enriquecer o que deve ser aprendido.

Pode ser incluído nessa dimensão como e quanto os alunos propositalmente se esforçam em fazer perguntas, oferecer uma sugestão ou contribuição, comunicar o que estão pensando e precisando, pedir esclarecimentos, gerar opções, comunicar gostos e desgostos, buscar maneiras de adicionar relevância pessoal ao conteúdo aprendido, e no casos dos alunos de Administração, ilustrar a teoria com casos reais, com o propósito de gerar contribuições construtivas para a sua própria aprendizagem.

Segundo Veiga (2013) esta dimensão liga-se a uma percepção do aluno como o agente da ação, e como as iniciativas deste aluno, intervenções nas aulas, diálogos com o professor, questões levantadas e sugestões feitas aos professores podem melhorar o processo de aprendizagem.

\section{ASPECTOS METODOLÓGICOS}

O estudo parte da pesquisa realizada por Veiga (2013), ao validar o instrumento de mensuração do envolvimento de alunos nas rotinas escolares sob quatro dimensões (cognitiva, afetiva, comportamental e agenciativa). Entretanto, o estudo foi realizado no ambiente de 
ensino superior, mais especificamente, nos Cursos de Ensino Superior em Administração que possuem instalações na cidade de Caxias do Sul. Por essa razão, o estudo se caracteriza por ser quantitativo descritivo.

A partir do instrumento validado, o questionário foi adaptado para questões do tipo escala Likert de sete pontos, variando de "discordo totalmente" a "concordo totalmente". E as palavras "escola" foram substituídas por "instituições". Para garantir a compreensão do questionário por parte dos respondentes, foi aplicado um pré-teste com 15 alunos.

A coleta de dados foi realizada em três diferentes instituições que oferecerem curso superior em Administração na cidade de Caxias do Sul, e que aceitaram participar do estudo. $\mathrm{Na}$ instituição A foram coletados 47 questionários e foram analisados 44 válidos (3 foram excluídos por não estarem completos). Já na instituição B foram coletados dados de 50 alunos e analisados 48 questionários válidos. Na instituição $\mathrm{C}$ foram coletados dados de 49 alunos e analisados 45 questionários válidos. A pesquisa contou com um total de 137 questionários válidos, dentre os 146 respondentes das três instituições.

A amostra se caracterizou pela maioria mulheres $(55,5 \%)$, residentes na cidade de Caxias do Sul (94,2\%), que iniciaram o curso de graduação entre 2000 e 2013. Em relação ao estágio do aluno no curso, percebe-se que a maioria dos alunos já se encontra com mais da metade do curso concluído. Mais de 59\% dos alunos afirmaram ter mais de 30 disciplinas concluídas.

Após a coleta, os dados foram tabulados e submetidos a técnicas estatísticas de análise descritiva e multivariadas, onde se realizou o teste ANOVA e Post Hoc, de acordo com Hair (2005). Os dados foram analisados com o auxílio do software IBM SPSS Statistics ${ }^{\circledR}$ versão 20 e planilha eletrônica.

\subsection{ANÁLISE DOS RESULTADOS}

Em um primeiro momento foi realizada uma análise de cada dimensão separadamente para cada instituição de ensino. A medida da adequação da amostra de Kaiser-Mayer-Olkin (KMO) analisa se a amostra está adequada à análise, ao comparar as magnitudes dos coeficientes de correlação observados e os coeficientes de correlação parciais (HAIR, 2005). Valores altos indicam que a análise é adequada. $\mathrm{Na}$ análise, os valores de $\mathrm{KMO}$ na dimensão cognitiva variaram entre 0,491 e 0,718 . $\mathrm{Na}$ dimensão afetiva tiveram resultados similares variando entre 0,626 e 0,685 . A dimensão agenciativa apresentou o mais baixo $\operatorname{KMO}(0,452)$ 
variando até 0,624 , finalmente a dimensão agenciativa apresentou o maior KMO para todas as instituições variando entre 0,717 e 0,858 . A Tabela 1 indica os fatores gerados com autovalor maior que 1 e o percentual de explicação acumulada a partir deles.

Tabela 1 Análise Fatorial

\begin{tabular}{|c|c|c|c|c|c|}
\hline Dimensões & $\begin{array}{c}\text { Quant. de } \\
\text { indicadores }\end{array}$ & Instituição & KMO & $\begin{array}{c}\mathbf{N}^{0} \text { de } \\
\text { Fatores }\end{array}$ & $\begin{array}{c}\text { \% de } \\
\text { explicação }\end{array}$ \\
\hline \multirow{3}{*}{$\begin{array}{l}\text { Dimensão } \\
\text { Cognitiva }\end{array}$} & \multirow{3}{*}{5} & Instituição A & 0,612 & 2 & 72,04 \\
\hline & & Instituição B & 0,718 & 1 & 47,40 \\
\hline & & Instituição C & 0,491 & 3 & 82,77 \\
\hline \multirow{3}{*}{ Dimensão Afetiva } & \multirow{3}{*}{5} & Instituição A & 0,685 & 2 & 82,34 \\
\hline & & Instituição B & 0,677 & 1 & 46,70 \\
\hline & & Instituição C & 0,626 & 2 & 76,97 \\
\hline \multirow{3}{*}{$\begin{array}{c}\text { Dimensão } \\
\text { Comportamental }\end{array}$} & \multirow{3}{*}{5} & Instituição A & 0,624 & 2 & 75,96 \\
\hline & & Instituição B & 0,452 & 2 & 62,25 \\
\hline & & Instituição C & 0,499 & 3 & 75,37 \\
\hline \multirow{3}{*}{$\begin{array}{c}\text { Dimensão } \\
\text { Agenciativa }\end{array}$} & \multirow{3}{*}{5} & Instituição A & 0,843 & 1 & 62,35 \\
\hline & & Instituição B & 0,717 & 1 & 59,05 \\
\hline & & Instituição C & 0,858 & 1 & 69,62 \\
\hline
\end{tabular}

Fonte: Elaborado pelos autores

Analisando a tabela 1, percebe-se que a dimensão agenciativa foi a que apresentou os menores valores de KMO e necessidade de mais fatores para explicar a variabilidade dos dados. Assim, pode-se inferir que na dimensão agenciativa a variabilidade é maior, para as três instituições de ensino. Após a avaliação da unidimesionalidade dos constructos através da análise fatorial procedeu-se a análise da matriz de correlações, na qual as dimensões foram correlacionadas, através da Correlação de Pearson, como apresentada na Tabela 2.

Tabela 2 Matriz de Correlação

\begin{tabular}{l|l|l|l|l}
\hline \multicolumn{1}{c|}{ Dimensões } & \multicolumn{1}{|c|}{$\begin{array}{c}\text { Dimensão } \\
\text { Cognitiva }\end{array}$} & $\begin{array}{c}\text { Dimensão } \\
\text { Afetiva }\end{array}$ & \multicolumn{1}{c}{$\begin{array}{c}\text { Dimensão } \\
\text { Comportamental }\end{array}$} & $\begin{array}{c}\text { Dimensão } \\
\text { Agenciativa }\end{array}$ \\
\hline Dimensão Cognitiva & 1 & & & \\
\hline Dimensão Afetiva &, $195^{*}$ & 1 & & \\
\hline Dimensão Comportamental &,$- 266^{* *}$ &, 112 & 1 & 1 \\
\hline Dimensão Agenciativa &, $348^{* *}$ &, $275^{* *}$ &,- 033 & \\
\hline *. Correlação é significante ao nível 0.05 (2-tailed). \\
**. Correlação é significante ao nível 0.01 (2-tailed)
\end{tabular}

Fonte: Elaborado pelos autores 
A matriz de correlações para as quatro dimensões indica que apesar de não apresentar valores altos de correlação, é possível perceber correlação significante $(s=0,00)$ positiva entre a dimensão agenciativa e cognitiva $(0,348)$. A dimensão agenciativa também apresentou correlação positiva significante $(\mathrm{s}=0,01)$ com a dimensão afetiva $(0,275)$. A dimensão cognitiva apresentou correlação significante $(s=0,02)$ com a dimensão comportamental de forma negativa $(-0,266)$ o que demonstra baixa correlação entre as dimensões. Em contraponto a dimensão cognitiva apresentou correlação significativa $(0,023)$, mas positiva com a dimensão afetiva $(0,195)$ o que indica correlação, mesmo que maneira fraca, entre as duas dimensões.

A partir da análise de correlação das dimensões procedeu-se análise das médias que os alunos atribuíram a cada dimensão em cada uma das instituições. A tabela 3 apresenta os valores da média e desvio padrão para cada dimensão e instituição.

Tabela 3 Média e Desvio Padrão de cada dimensão por instituição

\begin{tabular}{l|c|c|c|c|c|c} 
& \multicolumn{2}{|c|}{ Instituição A } & \multicolumn{2}{c}{ Instituição B } & \multicolumn{2}{c}{ Instituição C } \\
\hline \multicolumn{1}{c|}{ Dimensões } & Média & $\begin{array}{c}\text { Desvio } \\
\text { Padrão }\end{array}$ & Média & $\begin{array}{c}\text { Desvio } \\
\text { Padrão }\end{array}$ & Média & $\begin{array}{c}\text { Desvio } \\
\text { Padrão }\end{array}$ \\
\hline Cognitiva & 4,40 & 0,99 & 4,70 & 0,88 & 5,06 & 0,65 \\
\hline Afetiva & 3,64 & 0,77 & 3,70 & 0,61 & 4,00 & 0,60 \\
\hline Comportamental & 1,91 & 1,07 & 1,80 & 0,76 & 1,59 & 0,56 \\
\hline Agenciativa & 3,19 & 1,24 & 4,02 & 1,35 & 4,47 & 1,24
\end{tabular}

Fonte: Elaborado pelos autores

$\mathrm{Na}$ tabela 3 é possível perceber que a Instituição apresentou em três das quatro dimensões analisadas a maior média (Cognitiva, Afetiva e Agenciativa). Entretanto a dimensão comportamental da instituição $C$ apresentou a menor média $(1,59)$ em relação a todas as outras instituições. A instituição A e B apresentaram médias similares nas dimensões afetiva (3,64 e 3,7 respectivamente) e comportamental (1,91 e 1,80 respectivamente). Nas dimensões cognitiva e agenciativa a diferença entre a instituição A e B também se mantiveram próximas.

É possível perceber que a dimensão comportamental apresentou a menor média em todas as instituições, atingindo médias abaixo de 2 em todos os casos, podendo ser considerada baixa, uma vez que se posiciona próximo do termo Discordo Totalmente . A dimensão que apresentou a maior média em todas as instituições foi a dimensão cognitiva logo seguida pela dimensão agenciativa. 
Na sequência foi realizada a análise de variância, ou ANOVA, tendo em vista ser um procedimento estatístico utilizado para determinar se as diferenças entre as médias de três ou mais grupos de dados são estatisticamente significativos (HAIR, 2005). Para atender o objetivo do trabalho de entender o envolvimento dos alunos de diferentes instituições de ensino superior em Administração, em relação às dimensões cognitiva, afetiva, agenciativa e comportamental foram desenvolvidas as hipótese nula e alternativa, para posteriormente serem confirmadas ou rejeitadas na análise dos dados.

Hipótese Nula: O envolvimento dos alunos do Curso de Graduação em Administração nas quatro dimensões são os mesmos, independente da instituição.

Hipótese Alternativa: Existe diferença em relação ao envolvimento dos alunos em relação às instituições de ensino do Curso de Graduação em Administração.

Definidas as hipóteses se realizou a análise de variância, demonstrada na Tabela 4, onde é possível perceber os valores da tabela ANOVA assim como a significância do teste para cada uma das dimensões.

Tabela 4 ANOVA das dimensões

\begin{tabular}{l|l|c|c|c|c|c}
\hline \multicolumn{2}{l|}{} & SQ & GL & QM & F & Sig. \\
\hline \multirow{3}{*}{$\begin{array}{l}\text { Dimensão } \\
\text { Cognitiva }\end{array}$} & Entre Grupos & 9,676 & 2 & 4,838 & 6,712 &, 002 \\
\cline { 2 - 7 } & Dentro dos Grupos & 96,584 & 134 &, 721 & & \\
\cline { 2 - 7 } $\begin{array}{l}\text { Dimensão } \\
\text { Afetiva }\end{array}$ & Total & 106,260 & 136 & & & \\
\hline \multirow{3}{*}{$\begin{array}{l}\text { Dimensão } \\
\text { Comportamental }\end{array}$} & Entre Grupos & 3,439 & 2 & 1,720 & 3,923 &, 022 \\
\cline { 2 - 7 } & Dentro dos Grupos & 58,732 & 134 &, 438 & & \\
\cline { 2 - 7 } & Total & 62,171 & 136 & & &, 165 \\
\hline \multirow{3}{*}{$\begin{array}{l}\text { Dimensão } \\
\text { Agenciativa }\end{array}$} & Entre Grupos & 2,476 & 2 & 1,238 & 1,828 & \\
\cline { 2 - 7 } & Dentro dos Grupos & 90,743 & 134 &, 677 & &, 000 \\
\cline { 2 - 7 } & Entre Grupos & 93,219 & 136 & & & \\
\cline { 2 - 7 } & Dentro dos Grupos & 219,468 & 134 & 1,638 & & \\
\cline { 2 - 7 } & Total & 256,969 & 136 & & & \\
\hline
\end{tabular}

Fonte: Elaborado pelos autores

Para a comparação de médias entre os três grupos, os elevados valores de F, principalmente nas dimensões agenciativa $(F=11,449, \mathrm{p}<0,001)$ e cognitiva $(F=6,712$, $\mathrm{p}=0,002)$, indicam que há diferença entre esses grupos. A dimensão afetiva também indicou diferenças significativas entre as instituições, porém com valor mais baixo de significância $(p=0,022)$. Embora a análise de variância identifique diferenças entre os grupos, ela não 
permite apontar com precisão as diferenças significantes entre as instituições de ensino. Para obter informações adicionais, sobre aonde essas variáveis diferem se realizou o teste de comparação das médias de Tukey (Tabela 5) para as instituições e em cada uma das dimensões estudadas.

Tabela 5 Teste de comparação de médias

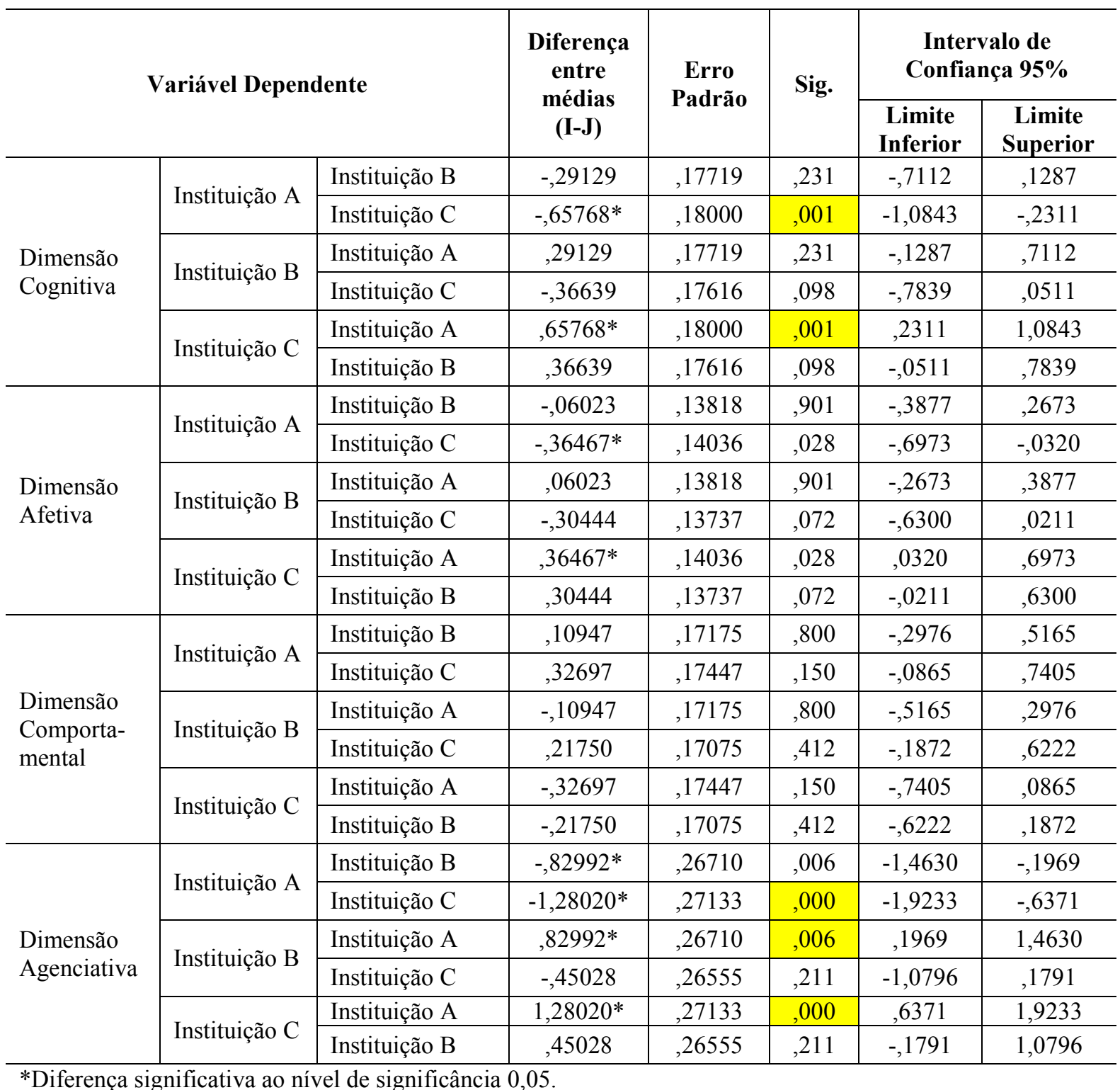

*Diferença significativa ao nível de significância 0,05 .

Fonte: Elaborado pelos autores

No teste é possível confirmar a rejeição da hipótese nula ao perceber diferenças significantes sobre o envolvimento dos alunos do Curso de Graduação em Administração em três das quatro dimensões. 
Ao analisar a dimensão agenciativa, que na ANOVA apresentou o valor de $\mathrm{F}$ mais elevado $(\mathrm{F}=11,449)$, foi possível perceber que a instituição $\mathrm{A}$, que apresentou a menor média entre as instituições nesta dimensão $(3,2)$ teve diferença significativa em relação a Instituição $\mathrm{B}($ dif $=0,82992)$ e da instituição $C($ dif $=1,28020), A$ instituição $B$ e C não demonstraram diferenças significantes nesta dimensão.

A dimensão cognitiva apresentou as maiores médias em torno de 4,7 sendo os alunos da instituição C aqueles com maior média. Na ANOVA apresentou o segundo mais alto valor de $F(F=6,712)$, apresentando diferença significante entre os alunos da Instituição A e C (dif $=0,65768)$ o que indica diferença entre esses grupos. Não foram identificados valores significativos na relação a Instituição A e B, nem na relação da Instituição B e C.

$\mathrm{Na}$ dimensão afetiva que na análise da ANOVA apresentou valor de F significante ( $\mathrm{F}$ $=3,923$ ), foi possível perceber diferença entre os alunos da Instituição A e C (dif = 0,36467). No entanto não foram identificadas diferenças significativas na relação da Instituição A e B, nem na relação da Instituição B e C. Assim como nas dimensões cognitiva e agenciativa, na dimensão afetiva a instituição $\mathrm{C}$ apresentou valores mais altos.

\section{DISCUSSÃO}

Este trabalho apresentou como objetivo do trabalho entender e distinguir o envolvimento dos alunos que cursam Administração, em três diferentes instituições de ensino superior em Administração da Cidade de Caxias do Sul. Foram levadas em consideração quatro dimensões específicas: cognitiva, afetiva, comportamental e agenciativa, definidas a partir da literatura e instrumentos validados, com o propósito de entender se existem diferenças/similaridades na relação da instituição com envolvimento dos alunos e como elas se comportam.

Através da análise dos dados foi possível rejeitar hipóteses que o envolvimento dos alunos do Curso de Graduação em Administração nas quatro dimensões são os mesmos, independente da instituição. Por consequência acabou confirmando a segunda hipótese que trata de diferenças em relação ao envolvimento dos alunos dentre as diferentes instituições de ensino superior em Administração de Caxias do Sul.

Analisando cada uma das dimensões relacionadas às instituições, na dimensão Cognitiva, se observou como as estratégias adotadas pelos alunos utilizadas no processo de aprendizagem poderia melhorar o desempenho acadêmico (WALKER, GREENE e 
MANSELL, 2006; LAM et al., 2011; VEIGA 2013). Pode-se perceber diferença significante entre as instituições analisadas, quando se fala no processamento e gestão da informação, principalmente entre duas instituições específicas.

As mesmas instituições que mostraram divergências na dimensão Cognitiva, também apresentaram diferenças na dimensão Afetiva, entretanto com menor significância. A dimensão afetiva se refere aos sentimentos do aluno sobre o processo, contexto e pessoas envolvidos na aprendizagem (CONNELL e WELLBORN, 1991; LAM et al., 2011) sendo diretamente ligado ao senso de pertencimento à instituição (FINN, 1989).

A dimensão que apresentou maior divergência entre as respostas foi a dimensão Agenciativa. Esta, definida por Reeve e Tseng (2011), como o engajamento do aluno para que de forma intencional e pró-ativa ele busca personalizar e enriquecer o aprendizado, foi a que mais demonstrou diferença entre as instituições. Ou seja, cada grupo entende de maneira diferente a maneira agenciativa de fomentar o aprendizado.

Por outro lado, a dimensão comportamental citada por Archambault et al. (2009), que reflete diretamente nas dimensões cognitivas, mas que leva as características psicológicas em consideração, e as atribui a questões básicas de participação e de conformidade dos alunos no processo de aprendizagem, não apresentaram diferença estatística, ou seja, houve um tendência de respostas similares entre os grupos. Uma possibilidade que explicaria a ausência de diferença entre os grupos no aspecto comportamental é que todos fazem parte de um mesmo município é o ambiente cultural da região pode ter influencia no comportamento destes alunos. Outra possibilidade de explicação desta dimensão é que o fato de quase $60 \%$ dos alunos já concluíram mais 30 disciplinas do curso, o que demonstra que estes alunos já estão com maturidade acadêmica em relação ao curso, já que se encontra em fase de conclusão. Outro ponto que pode também ser destacado na dimensão comportamental, é que a apresentação da menor média em comparação as demais instituições, é que ao se levar se em consideração que os indicadores da dimensão comportamental retratados por Veiga (2013), abordam comportamentos de alunos nos ambientes escolares, tais como perturbar intencionalmente as aulas, o ser incorreto com os professores, o estar distraído nas aulas e, ainda, o faltar às aulas, o resultado média baixa (menor que 2) demonstra que o comportamento dos alunos tende a não concordar com as afirmações feitas na escala, já que se aproximam do termo Discordo Totalmente.Tendo em vista que três das quatro dimensões apresentaram diferenças entre as instituições, se percebe que o contexto das instituições pode 
exercer influência sobre as dimensões analisadas, impactando no aprendizado do aluno pois mudam as estratégias de aprendizagem, seus sentimentos e a forma como ele atua como agente da vida acadêmica. Estudos nesta natureza podem contribuir para instituições consigam acompanhar o processo de aprendizagem dos alunos muito além do desempenho acadêmico.

\section{AGRADECIMENTOS}

Fundação de Amparo a Pesquisa do Rio Grande do Sul - FAPERGS/CAPES

\section{REFERENCIAS}

ABRES - Associação Brasileira de Estágios. Disponível em $<$ http://www.abres.org.br/v01/stats/>. Acesso em julho de 2014.

ARCHAMBAULT, I., JANOSZ, M., FALLU, \& J. \& PAGANI, L. S. (2009). Student engagement and its relationship with early high school dropout. Journal of Adolescence 32, $651-670$.

AUSTIN, Ann E. The Work Experience of University and College Administrators. Administrator's Update, v. 6, n. 1, p. n1, 1984.

CONNELL, James P.; WELLBORN, James G. Competence, autonomy, and relatedness: A motivational analysis of self-system processes. 1991.

ENGERS, Maria Emília Amaral; MAROSINI, Marília Costa. Pedagogia universitária e aprendizagem. EDIPUCRS, 2007.

FELICETTI, Vera Lucia; MOROSINI, Marília Costa. Do compromisso ao comprometimento: o estudante ea aprendizagem. Educar em Revista, v. 2, 2010.

FIOR, Camila Alves; MERCURI, Elizabeth; SILVA, Dirceu da. Evidências de validade da Escala de Envolvimento Acadêmico para universitários.Avaliação Psicológica, v. 12, n. 1, p. 81-89, 2013.

HAIR, J. F. Análise Multivariada de Dados. Bookman, 2005.

IBGE - Instituto Brasileiro de Geografia e Estatística. Disponível em

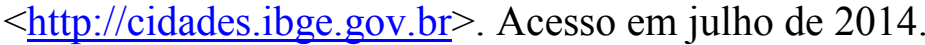

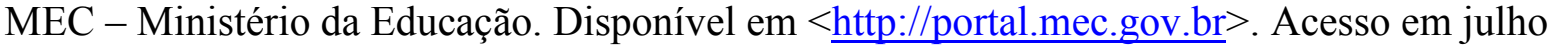
de 2014. 
LAM, Shui-fong et al. Do girls and boys perceive themselves as equally engaged in school? The results of an international study from 12 countries.Journal of school psychology, v. 50, n. 1, p. 77-94, 2012.

MILLER, K. A. Intrinsic Motivation and Self-Determination in Human-Behavior-Ryan, RM. 1988.

NILSEN, Hallgeir. Influence on student academic behaviour through motivation, self-efficacy and value-expectation: An action research project to improve learning. Issues in Informing Science and Information Technology, v. 6, p. 545-556, 2009.

PASCARELLA, Ernest T.; TERENZINI, Patrick T. How college affects students. San Francisco: Jossey-Bass, 2005.

REEVE, Johnmarshall; TSENG, Ching-Mei. Agency as a fourth aspect of students' engagement during learning activities. Contemporary Educational Psychology, v. 36, n. 4, p. $257-267,2011$.

SKINNER, Ellen A.; BELMONT, Michael J. Motivation in the classroom: Reciprocal effects of teacher behavior and student engagement across the school year. Journal of educational psychology, v. 85, n. 4, p. 571, 1993.

VEIGA, Feliciano. Envolvimento dos alunos na escola: Elaboração de uma nova escala de avaliação. 2013.

WALKER, Christopher O.; GREENE, Barbara A.; MANSELL, Robert A. Identification with academics, intrinsic/extrinsic motivation, and self-efficacy as predictors of cognitive engagement. Learning and Individual Differences, v. 16, n. 1, p. 1-12, 2006. 